\title{
Raman LIDAR for UHECR experiments: an overview of the L'Aquila (Italy) lidar station experience for the retrieval of quality-assured data
}

\author{
Marco Iarlori ${ }^{1, *}$, Vincenzo Rizi ${ }^{1}$, Giuseppe D’Amico ${ }^{2}$, Volker Freudenthaler $^{3}$, Ulla Wandinger $^{4}$, and Aurelio Grillo ${ }^{1}$ \\ ${ }^{1}$ CETEMPS and INFN, Dipartimento di Scienze Fisiche e Chimiche, Università degli Studi dell'Aquila, via Vetoio, 67100, L'Aquila, \\ Italy \\ ${ }^{2}$ Consiglio Nazionale delle Ricerche - Istituto di Metodologie per l'Analisi Ambientale (CNR-IMAA), Contrada S. Loja - Zona \\ Industriale, 85050 Tito Scalo (Potenza), Italy \\ ${ }^{3}$ Ludwig-Maximilians-Universität, Meteorologisches Institut Experimentelle Meteorologie, Munich, Germany \\ ${ }^{4}$ Leibniz Institute for Tropospheric Research, Permoserstr. 15, 04318 Leipzig, Germany
}

\begin{abstract}
L'Aquila (Italy) lidar station is part of the EARLINET (European Aerosol Research Lidar Network) since its beginning in the 2000. In the EARLINET community great efforts are devoted to the quality-assurance of the aerosol optical properties inserted in the database. To this end, each lidar station performed intercomparisons with reference instruments, a series of internal hardware checks in order to assess the quality of their instruments and exercises to test the algorithms used to retrieve the aerosol optical parameters. In this paper we give an overview of our experience within EARLINET qualityassurance (QA) program, which was adopted for the Raman lidar (RL) operated in the AUGER Observatory. This program could be systematically adopted for the lidar systems needed for the current and upcoming UHECR experiments, like CTA (Cherenkov Telescope Array).
\end{abstract}

\section{Introduction}

The EARLINET (European Aerosol Research Lidar Network, www.earlinet.org) was founded in year 2000, involving 19 lidar systems located in 11 countries, with the main purpose of establishing an aerosol climatology for Europe. EARLINET community has grown and it currently consists of 35 lidar systems in 16 countries [1]. At beginning, the EARLINET lidar systems consisted mainly in single wavelength UV Raman lidars, like the one that is operated in L'Aquila [2], while nowadays a lot of them have been upgraded to the so-called $3+2+\delta$ Raman lidars. The expression $3+2+\delta$ means that these modern Raman lidars are able to retrieve independently the profiles of the backscatter coefficients at $355 \mathrm{~nm}, 532 \mathrm{~nm}$ and $1064 \mathrm{~nm}$ wavelengths, and of 2 aerosol extinction coefficients at $355 \mathrm{~nm}$ and $532 \mathrm{~nm}$, and of the aerosol linear depolarization ratio [3]. The multi-wavelength capability allows to retrieve the aerosol microphysical properties as well [4].

The lidar systems in EARLINET are very different each other being the instruments designed and assembled in-house; the same is true for the retrieval algorithms, anyway the EARLINET community is making significant improvements to uniform the retrieval of the aerosol optical parameter profiles developing the Single Calculus Chain [5].

The different lidar systems are continuously upgraded, and for these reasons, the EARLINET community adopted an intensive quality-assurance (QA) program. That QA program was improved in the successive collaborations like EARLINET-ASOS (Advanced Sustainable Observation System) and ACTRIS (Aerosols, Clouds, and Trace gases Research InfraStructure), and it is still operative in the framework of ACTRIS-2 research infrastructure project.

We briefly describe the EARLINET QA program and, as an example, some of the results related to L'Aquila RL.

Finally, we propose to adopt the QA program activities for the lidar systems needed to UHECR experiments.

\section{The EARLINET QA program}

The EARLINET QA program at instrument level consists of:

- intercomparison campaigns with a reference lidar system [6];

- applying internal system check-up tools, developed to perform tests on regular basis and/or each time the lidar system is substantially changed [7].

At software level, several exercises have been prepared to test:

\footnotetext{
Corresponding author: marco.iarlori@aquila.infn.it
} 
- the algorithm to retrieve the profile of the backscatter coefficient from elastic-backscatter lidar signals [8];

- the algorithm to retrieve the profiles of the backscatter and extinction coefficient from RL signals [9].

\subsection{Lidar systems Intercomparison}

The first EARLINET lidar intercomparison campaign was performed in 2000 and was based on a previous successful experience in the framework of the German Aerosol Lidar Network [10]. In this campaign it was found that, among the lidar systems, the typical mean deviations of aerosol backscatter coefficients in both the planetary boundary layer and free troposphere were within the predefined quality criteria $[11,6]$.

Other campaigns were performed by comparing the data taken with each lidar system to the ones simultaneously measured with a co-located reference lidar.

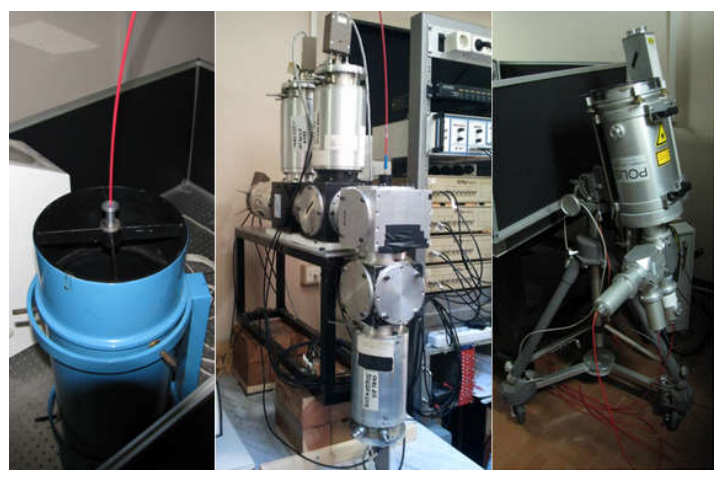

Fig. 1. Left and central panel: the L'Aquila RL receiver. Right panel: the reference lidar system for the 2012 intercomparison campaign, i.e. the POLIS (Portable Lidar System) system of the Ludwig-Maximilians-Universität, Meteorological Institute, Munich, Germany.

Because of the substantial upgrade of the lidar systems, the most recent intercomparison campaigns involved both elastic and Raman channels and, as consequence, the comparison of the aerosol backscatter and extinction profiles. The raw lidar signals were also compared to check for system problems, their causes and possible solutions [6].

These recent campaigns evidenced that most of the EARLINET lidar systems performs well, for example, the mean relative systematic signal deviations from the reference lidar system were found to be within 1-2\%. The comparison of the aerosol optical properties has shown that the aerosol backscatter could be retrieved with an accuracy better than $\pm 2 \times 10^{-4} \mathrm{~km}^{-1} \mathrm{sr}^{-1}$ and the extinction better than $\pm 0.01 \mathrm{~km}^{-1}$.

The L'Aquila lidar system performed quite well in all the intercomparison campaigns. For this reason, it was used as reference for the Auger Observatory RL [12].

\subsection{Hardware check-up tools}

Part of the instrumental QA is constituted by a number of tools. These tools are useful to test the performances of lidar sub-systems, i.e. telescope-laser alignment. EARLINET lidar stations have to perform these tests on regular basis, These tools are fully documented [7] and we give only a brief overview of their use.

\subsubsection{Telecover test}

There is no calibration method for a lidar system in the near range, where clean air conditions are almost never met. The near range is also where the receiver optical performances could have the greatest impact.

In the telecover test [13], the backscattered photons collected from different sectors of the telescope are compared. In order to perform these measurements, the telescope aperture is covered leaving open only a defined sector at time (north $(\mathrm{N})$, south $(\mathrm{S})$, east $(\mathrm{E})$ and west (W) quadrants, see Fig. 2). In the ideal case these signals have to exhibits no difference, but the overlap range. Measurements with full telescope aperture are done to check if the atmospheric condition can be considered stable during the test.

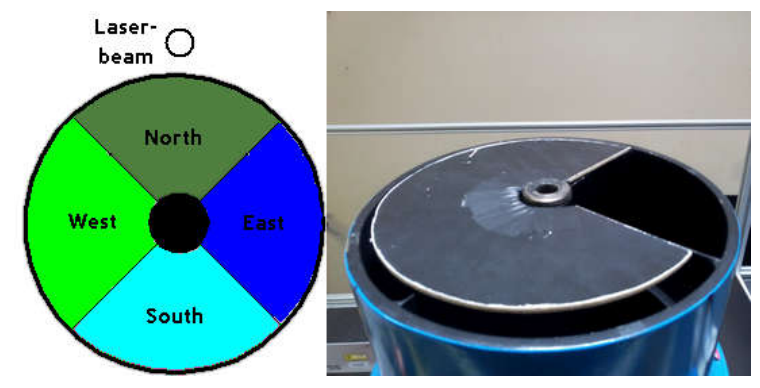

Fig. 2. On the left panel it is shown the nomenclature of the quadrants for the telecover test. On the right panel, the primary mirror during the test.

If range dependent differences are spotted, a further inspection to find out the possible causes should be made (for example misalignments or spatial inhomogeneity of the detector sensitivity, should be investigated). It could be also useful to have a ray tracing simulations of the lidar receiver.

The mean signal $(\mathrm{M})$ is the average of the signals returns in each sector: $\mathrm{M}=(\mathrm{N}+\mathrm{E}+\mathrm{S}+\mathrm{W}) / 4$, then the relative deviations could be calculated as: $\mathrm{Ndev}=(\mathrm{N}-$ $\mathrm{M}) / \mathrm{M}$ for each sector. The overall relative deviation is: AllDev $=\operatorname{sqrt}\left[\left(\mathrm{Ndev}^{2}+\mathrm{Edev}^{2}+\mathrm{Sdev}^{2}+\mathrm{Wdev}^{2}\right) / 4\right]$.

Internal quality criteria [7] related to the values of the relative deviations help to qualify the alignment and the optical performances of the lidar system. L'Aquila RL has fulfilled these criteria along all the performed tests.

\subsubsection{Rayleigh fit test}

While the telecover test checks the lidar system performance in the near range, the Rayleigh fit test is the check-up tool for the system behaviour in the far range i.e. in the free troposphere and beyond. In these regions 
are likely the presence of aerosol-free atmospheric layers and is possible to measure the agreement between a calculated pure molecular signal and a real lidar signal.

Basically, the Rayleigh fit is a normalization of a lidar signal to the calculated attenuated Rayleigh backscatter coefficient in an aerosol free region. EARLINET internal criteria for the quality of the fit are based on statistical analysis of the fit residuals [7]. An evaluation "by eye" of the fit by a lidar expert is sufficient to recognize the more evident signal distortions, not reported during the tests on the L'Aquila $\mathrm{RL}$, and then investigate for possible causes (e.g. misalignment).

\subsubsection{Zero-bin test}

If the delay between the laser pulse emission and the start of the acquisition system is not well known, or in other words, if the range scale is not correctly set, there could be a quite large indetermination in the retrieval of the aerosol extinction in the near range, especially in the very first kilometer. This range dependent absolute value of the extinction indetermination $(\Delta \alpha)$ could be calculated as function of the uncertainty $\left(\mathrm{r}_{0}\right)$ in the position of the true zero-range [7]:

$$
\Delta \alpha\left(r, r_{0}\right)=\frac{2}{1+\left(\frac{\lambda_{\text {Raman }}}{\lambda_{\text {Laser }}}\right)^{k}} \frac{1}{\left(r-r_{0}\right)^{2}} r_{0} .
$$

In Eq.(1), $r$ is the range, $\lambda_{\text {Laser }}$ is the laser wavelength, $\lambda_{\text {Raman }}$ is the wavelength of the $\mathrm{N}_{2}$ Raman backscattered photons, and $k$ is the Ångström exponent, which depend mainly on the aerosol size and it could be set to 1 for most of the aerosol in the planetary boundary layer (PBL). In this condition, if the resolution of the acquisition system is $15 \mathrm{~m}$ and the zero-range uncertainty $r_{0}$ is of just one range bin, the absolute value $\Delta \alpha$ at $355 \mathrm{~nm}$ is about $0.1 \mathrm{~km}^{-1}$ at $0.4 \mathrm{~km}$ range and it became $<0.01 \mathrm{~km}^{-1}$ only above $1 \mathrm{~km}$ range. Then also for a relatively small $r_{0}$ value, $\Delta \alpha$ could be of the same order of the typical extinction value in the PBL.

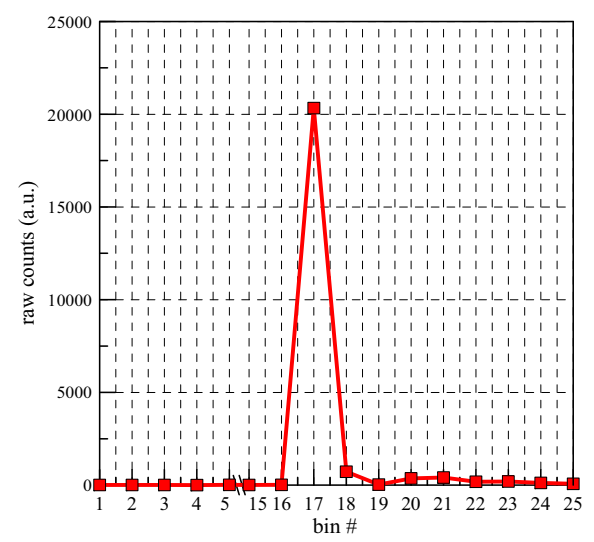

Fig. 3. Zero-bin measurement for the L'Aquila UV RL. Pretriggering allows to measure the first range gate associated to the start of the laser pulse (the $17^{\text {th }}$ bin).
Pre-trigger are often set in the acquisition of a lidar system, i.e. the acquisition starts to record before the actual time of the laser pulse emission. In this condition the zero-bin test can be performed: the lidar system can measure the photons reflected by a nearby obstacle (i.e., into laboratory). A quite definite peak is usually recorded, and it marks the location of the zero-bin, i.e. the actual start of the range scale (Fig.3).

This test has been performed on the RL build for the Auger Observatory [12]. Because the UHECR measurement sites are particularly clean from point of view of the aerosols content, an accurate zero-bin measurement is important to decrease the magnitude of the systematic uncertainty in the aerosol optical depth estimation.

\subsubsection{Dark measurement}

A dark measurement is a standard lidar measurement performed with the telescope fully covered. In this way the signal acquired will contain no atmospheric backscattered photons, but only a signal due to interference sources, for example the various spurious pick-ups or other EM interferences synchronized with the laser trigger or any other instrumental distortion.

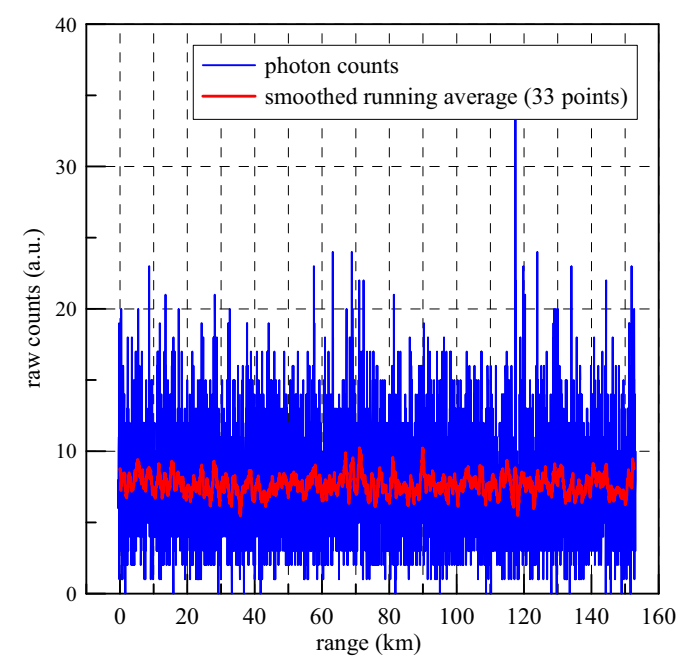

Fig. 4. An example of the dark measurement performed on the L'Aquila UV RL. It does not evidence any particular behavior.

Although currently there is no a standardized way to deal with the peculiar and not-stationary distortions that could be evidenced by this measurements, they are anyhow valuable because capable to point out the existence of hardware problems. The L'Aquila RL dark measurements do not show any distortions (Fig.4).

\subsection{Algorithms Intercomparison}

In the framework of EARLINET, several exercises have been carried out to ensure the quality of the algorithms for the retrieval of the aerosol optical parameter profiles. These tests on the algorithms were performed since the very start of the collaboration and all the results and the details can be found elsewhere $[8,9]$. 


\subsubsection{The aerosol backscatter inversion algorithm test (single elastic-backscatter lidar signal)}

The aim of this test was to investigate the implementation of the so-called Fernald inversion scheme [14] (or equivalent methods) in the algorithms used in each of the EARLINET stations. To this end, simulated elastic lidar signals were provided to the groups.

This blind test were organized in three different stages and each stage had several cases. In the first stage, the only additional information available was the atmospheric molecular density profile. In the second stage, the lidar ratio profile was provided and finally also the calibration parameters were made available. The first two stages provide the sensitivity to the input parameters, while the third stage was useful to actually test the goodness of the algorithms. The test was performed with simulated signals at $355 \mathrm{~nm}, 532 \mathrm{~nm}$ and at $1064 \mathrm{~nm}$.

The test evidenced the needs of an accurate estimation of the lidar ratio profile in order to retrieve a meaningful aerosol backscatter profile, especially for the UV signal. On the other hand, the retrieval at longer wavelengths evidenced stronger sensitivity to the calibration parameters.

The results for the third stage shown that all the EARLINET stations have a suitable algorithms for the retrieval of the aerosol backscatter profile.

This was also the case for the elastic-backscatter lidar inversion algorithm developed in L'Aquila, that in the third stage of the intercomparison produced profiles with an overall relative deviation from the true profile $<1 \%$ at all wavelengths [8].

\subsubsection{The aerosol backscatter and extinction inversion algorithm test (Raman lidar signals)}

The RL independently measures the aerosol extinction and backscatter profiles [15]. A non-scanning elastic lidar system is unable to do the same [16].

The retrieval algorithm for the extinction, has its key part in the numerical estimation of a derivative of the Raman return [9]; there are several methods to perform it, and most of them involve the use of smoothing filters. As a consequence of the filtering operation on the data, the range resolution of retrieved products become degraded [17].

The test of the Raman algorithms used in each of the EARLINET groups was blind and involved the algorithm for retrieval of the aerosol extinction profile starting from Nitrogen Raman signals, the algorithm for the retrieval of the aerosol backscatter and the lidar ratio estimation. The simulated Raman and elastic lidar signals were produced with a realistic aerosol profiles and noise.

The result of the algorithms tests are reported in Pappalardo et al. [9]. The mean deviation from the solution, for both the extinction and the backscatter profiles, have been found within the limits fixed in EARLINET [11]; for the lidar ratio, the mean relative deviations were found compatible with signal to noise ratio (SNR). The estimations of the statistical errors have been analyzed and they were consistent with the SNR embedded in the synthetic signals.
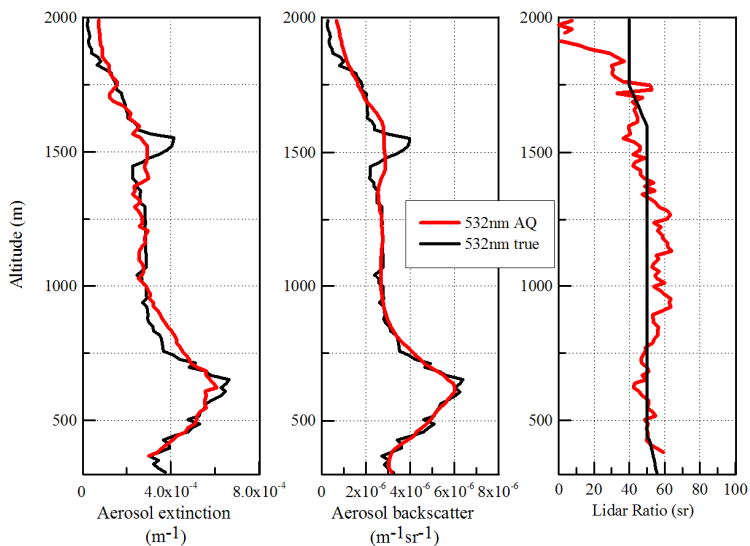

Fig. 5. The Raman algorithm test at $532 \mathrm{~nm}$ for the L'Aquila lidar station. Beside the general agreement between the true profiles (black lines) and the retrieved profiles (red lines), it can be noted how the smoothing filter embedded in the extinction retrieval algorithm, removed the peak at $1500 \mathrm{~m}$.

All the algorithms performed quite well, including the ones developed in L'Aquila, as it can be seen in the example reported in Fig.5. This figure also shows the importance of a correct use of the smoothing in order to get a consistent lidar ratio [17].

\section{Conclusions}

The key guidelines and methods of the intensive QA program developed by the EARLINET lidar community, have been briefly overviewed. The quality assurance is thought to be a dynamic process always ongoing, not just a one-time exercise.

It seems recommendable to adopt the ideas and concepts of the EARLINET QA program for the lidars built to be employed in UHECR experiments, in order to have data whose quality could be constantly traced.

As part of EARLINET, the L'Aquila RL has been successfully tested in hardware intercomparison campaigns, by continuative use of the internal hardware check-up tools, and in the quality checks of the inversion algorithms .

The L'Aquila lidar station, although is not one of the EARLINET reference systems, could be used as benchmarking system for lidars dedicated to the UHECR experiments. L'Aquila could also be considered as a source of certified algorithm codes for the retrieval of the profiles of the aerosol optical parameters.

Acknowledgements. The financial support for EARLINET in the ACTRIS Research Infrastructure Project by the European Union's Horizon 2020 research and innovation programme under grant agreement no. 654169 and previously under grant agreement no. 262254 in the 7th Framework Programme (FP7/2007-2013) is gratefully acknowledged. 


\section{References}

1. G. Pappalardo, et al., Atmos. Meas. Tech., 7, 23892409, doi:10.5194/amt-7-2389-2014 (2014).

2. V. Rizi, et al., Appl. Opt. 43, 6440-6453 (2004).

3. V. Freudenthaler, et al., Tellus B, 61, 165-179, doi: 10.1111/j.1600-0889.2008.00396.x, (2009).

4. D. Müller, et al., Atmos. Meas. Tech., 9, $5007-$ 5035, doi: 10.5194/amt-9-5007-2016 (2016).

5. G. D'Amico, et al., Atmos. Meas. Tech., 8, 48914916, doi: 10.5194/amt-8-4891-2015 (2015).

6. U. Wandinger, et al., Atmos. Meas. Tech., 9, 10011023, doi: 10.5194/amt-9-1001-2016 (2016).

7. V. Freudenthaler, et al., Atmos. Meas. Tech. Discuss., 2017 (in preparation).

8. C. Böckmann, et al., Appl. Opt. 43, 977-989 (2004).

9. G. Pappalardo, et al., Appl. Opt., 43, 5370-5385 (2004).

10. J. Bösenberg, et al., Rep. 317 Max-Planck-Institut für Meteorologie, Hamburg, Germany (2001).

11. V. Matthias, et al., Appl. Opt. 43, 961-976 (2004).

12. L. Wiencke, et al., Joint Elastic Side-Scattering Lidar and Raman Lidar Measurements of Aerosol Optical Properties in South East Colorado, submitted to J. Instrum.

13. V. Freudenthaler, The telecover test: a quality assurance tool for the optical part of a lidar system. Reviewed and Revised Papers Presented at the $24^{\text {th }}$ International Laser Radar Conference, Boulder, Colorado, USA, 23-27 June 2008, Boulder, Colorado, USA, presentation: S01P-30, available at: http://epub.ub. uni-muenchen.de/12958/ (2008) (last access: 10 November 2016).

14. F. G. Fernald, Appl. Opt. 23, 652- 653 (1984).

15. A. Ansmann, et al., Appl. Opt. 31, 7113-7131 (1992).

16. R. T. H. Collis and P. B. Russell, in Laser Monitoring of the Atmosphere (Springer-Verlag, Berlin, 1976) pp. 71-151.

17. M. Iarlori, et al., Atmos. Meas. Tech., 8, 5157-5176, doi: 10.5194/amt-8-5157-2015 (2015). 